\title{
ON VIBRATIONS OF L-SHAPED REGIONS
}

\author{
H.P.W. GOTTLIEB
}

School of Science, Griffith University, Nathan, Queensland 4111, Australia

(January 2001)

Number of pages: 5

Running title: L-shaped regions. 
The purpose of this paper is to point out that the numerical multi-domain method for free vibrations of membranes introduced by Kang and Lee [1] may be more accurate than they take credit for.

Consider their Case Study 4.3 of an L-shaped membrane (their Figure 10), constructed from three squares each of side length one unit. The normalized frequencies $\Lambda$ of a single such sub-square (wave speed one unit) are given exactly by

$$
\Lambda=\pi \sqrt{m^{2}+n^{2}} \quad, \quad \mathrm{~m}, \mathrm{n}=1,2,3, \ldots .
$$

But these constitute an analytical sub-class of the frequencies of the L-shape, and so may be used as standards for the accuracy of any numerical method for the L-shape for certain modes.

Inspection of modal patterns using any proprietary finite element package quickly reveals that the fundamental mode $m=n=1$ for the sub-square is unambiguously identifiable with the third sequential mode for the L-shape. The exact value $\pi \sqrt{ } 2 \approx 4.4429$ shows that the value 4.44 obtained for $\Lambda_{3}$ by Kang and Lee in [1] using their multi-domain method is actually better than the comparison value 4.45 obtained by Finite Element Methods quoted in [1].

Similarly, the eighth mode of the L-shape is identifiable as the second mode of the sub-square, with $m=1, n=2$, giving value $\pi \sqrt{ } 5 \approx 7.0248$ to which again the multidomain value 7.03 for $\Lambda_{8}$ obtained in [1] is closer than the FEM value 7.05 as quoted. In fact, the second and third modes of the sub-square, $m=1, n=2$ and $m=2, n=1$ in equation (1) above, are obviously degenerate. Correspondingly, the eighth and ninth modes of the L-shape are seen to be degenerate. It is a pity that the authors in [1] did not attempt just one further mode, which would presumably have detected this 
degeneracy. The detection of degeneracies and the degree of agreement between the computed corresponding eigenvalues may provide a further stringent test on the accuracy and efficacy of a numerical method.

Inspection of modal patterns for the L-shape shows that the $14^{\text {th }}$ mode corresponds to the fourth mode for the sub-square, with $m=n=2$ and eigenvalue $\pi \sqrt{ } 8 \approx$ 8.8858 . If the application in [1] could be extended up to $\Lambda_{14}$, this value would be a test of how the multi-domain method handles higher modes.

Although it has only been shown above that the multi-domain method results in [1] for the L-shape are in good agreement with exact results for an analytical subset of the modes (here specifically the $3^{\text {rd }}$ and the $8^{\text {th }}$ ), it seems likely that the numerical accuracy would persist for other multi-domain-method values listed in [1]. This is because the method of [1] involves only the Bessel function $\mathrm{J}_{0}$ so, from a numerical point of view in [1], there would be nothing special about the analytical modes whose eigenfunctions happen to involve trigonometric functions which make them amenable to an exact treatment.

These comments show the importance of having exact analytical modes to act as benchmarks for the accuracy of any proposed numerical method. Whilst such comparison modes may not be available for general shapes, their existence for some simple shapes may provide a class of test shapes. For example, if the L-shape consisted not of three identical squares but had sides in low-order rational ratios, some analytical modes could still be found, although they would have higher sequential mode numbers within the eigenvalue spectrum. It was fortunate that the simple L-shape considered in [1] had an exact mode as low as the third.

In conclusion, it may be noted that these ideas are also applicable to the numerical methods used for calculating the eigenfrequencies of simply-supported 
plates of polygonal shapes, via the membrane-plate analogy which relates the frequencies of the plate to the squares of the frequencies of a membrane of the same shape. (See e.g. the Appendix of [2] and references therein.)

For instance, R. Solecki [3] investigated the vibrations of a simply-supported L-shaped plate, regarded as a square plate with a square cutout, using a Boundary Integral Equation method. The third mode (for cut-out ratio 0.50 in [3]) should again correspond to $\mathrm{m}=\mathrm{n}=1$ in equation (1) above, but now $\Lambda$ is squared. With appropriate normalizations, this gives $\left(1^{2}+1^{2}\right) /(1 / 2)^{2}=8$ for the standard L-shape described above. And indeed, Mode 3 for this case was computed in [3] to have the very close value 8.001. Interestingly, the finite element program used as a comparison in [3] for the results produced a value 8.015 , again less accurate than Solecki's value according to the criterion stated above. 


\section{REFERENCES}

1. S.W. Kang and J.M. Lee 2000 Journal of Sound and Vibration 234, 455-470. Application of free vibration analysis of membranes using the non-dimensional dynamic influence function. doi:10.1006/jsvi.1999.2872

2. H.P.W. Gottlieb and J.P. McManus 1998 Journal of Sound and Vibration 212, 253-264. Exact shared modal functions and frequencies for fixed and free isospectral membrane shapes formed from triangles. doi:10.1006/jsvi.1997.1436

3. R. Solecki 1997 Journal of Vibration and Acoustics (Transactions of the ASME) 119, 464-467. Vibration of a simply supported L-shaped plate. 